\title{
A Comparison of Concept Selection in Concept Scoring and Axiomatic Design Methods
}

\author{
Adrian Xiao, Simon S. Park, Theo Freiheit \\ Department of Mechanical and Manufacturing Engineering \\ Schulich School of Engineering, University of Calgary, Canada \\ simon.park@ucalgary.ca
}

\begin{abstract}
The appropriate selection of design concepts has a strong influence on product cost, durability, robustness and functionality. Effective tools to identify good design concepts are critical. Different design methodologies have different objectives to aid in making the right decision. Some design methods are complementary, whereas others may provide contradictory results. In this study, two design methods, concept scoring and axiomatic design, are compared for their ability to obtain good design concepts. Conceptual designs of micro-pumps, which provide pressure gradients to actuate the flow of liquids and gas, are used to evaluate the design methods. Metrics are used to compare these two design methodologies' abilities to achieve objective evaluations. The concept scoring method is easy to use, especially when comparing different designs. The axiomatic design method provides a structured and mathematical design evaluation; however, achieving good design through the uncoupling or decoupling of matrices is challenging. Further study is required to integrate these two design methods.
\end{abstract}

\section{Introduction}

Concept, or early stage, design is considered to be the most difficult, sensitive and critical design in creating products [1]. It greatly influences the cost, robustness, manufacturability, and development time of the final products [2]. As a rule of thumb, the cost of engineering changes increases by ten times when changes are made in a later stage. Concept selection is the decision-making phase of concept design, where designers evaluate concepts with respect to customer needs and the designers' intention. This stage also includes comparing the relative strengths and weaknesses of the concepts, and determining concepts for further investigation, testing or development [1].
This phase significantly affects whether the final design will be exemplary, resulting in higher profits, or will have fundamental flaws that will often require costly recalls. Many designers are challenged during this process, by having to make many decisions with limited information. Many design methods [3] have been developed to assist designers in making the correct decisions during the concept selection phase, including Pugh's concept scoring [4], the analytic hierarchy process (AHP), fuzzy set [5], the fuzzy AHP method [6], flexible design concept selection [7], the hypothetical equivalents and in-equivalents method [8], quality function deployment (QFD) [9], and axiomatic design [12].

Among these methods, the concept scoring and AHP methods are most commonly used in industry [10]. In essence, concept scoring and the AHP method have fundamentally the same methodologies. The concept scoring method is a numerically weighted Pugh method, while AHP is a more mathematic version of concept scoring that minimizes personal bias during the weighting and scoring. According to Yeo et al. [6], concept scoring and AHP have been found to provide similar results, as long as the differentiation between the alternatives is sufficiently large. Furthermore, the simplest and most commonly used form of concept selection methods has also been found to be the most applicable to supporting the fuzzy nature of concept selection [6]. However, limitations, such as uncertainty [11] and inability to provide the information on the compatibility between design concepts [7], remain as challenges for the concept selection method.

Contrast to the concept scoring and AHP methods, the axiomatic design method [12] provides two design axioms that present the necessary tools for the evaluation of good or bad designs. According to $\mathrm{Hu}$ and Pieprzak [13], the fundamental set of principles that define good axiomatic design practice can help to facilitate the generation of good design concepts. 
In this paper, the abilities of both formal concept scoring and axiomatic design methods to select design concepts are evaluated through three concept designs of micro-pumps. Eight concept scoring and seven axiomatic design criteria are proposed and used to evaluate the design concepts. In order to compare the two methods, six metrics are generated to identify strengths and weaknesses. The objectives of this paper are to find the strengths, weaknesses, and complementary aspects of the concept scoring and axiomatic design methods, and to propose a new approach for concept selections through joint use of the two methods.

\section{Approach}

The approach taken in this paper is shown in Figure 1. First, the design requirements are collected and decomposed into the functional requirements. Then, the concept solutions are generated based on the decomposed functional requirements. Next, design concepts are produced, based on possible concept solutions. Once the concept designs are obtained, both the concept scoring and axiomatic design methods are used to evaluate these design concepts. Finally, the two design methods are compared and assessed, with respect to concept selection ability.

\subsection{Functional Decomposition and Concept Design}

Although design functions do not have a clear, uniform, objective, and widely accepted definition, it is generally agreed that they are the most important aspect in determining a product's basic characteristics [14]. The product or functional requirements are usually generated based on a designer's understanding of the product and its market.

Functional decomposition is the process of redefining the product requirements into sub-functions, so that a solution for all the sub-functions can be envisioned by the designer. The functional decomposition method is like going to a supermarket where the necessary ingredients for the end result are selected. Different designers may develop different functional decompositions, since functional decomposition does not follow just one recipe; however, it provides good insight into available options. Clearly defined and decomposed design requirements help the generation and selection of solution technologies, i.e. the operating principles of the design.

A traditional methodology for function decomposition is the representation of the total functions and subfunctions where the inputs are information, material and energy, and the output is desired outcomes as shown in Figure 2. The total function of a product consists of several sub-functions. Different designers may develop different functional decompositions [1] depending on the market, experience, costs and availability.

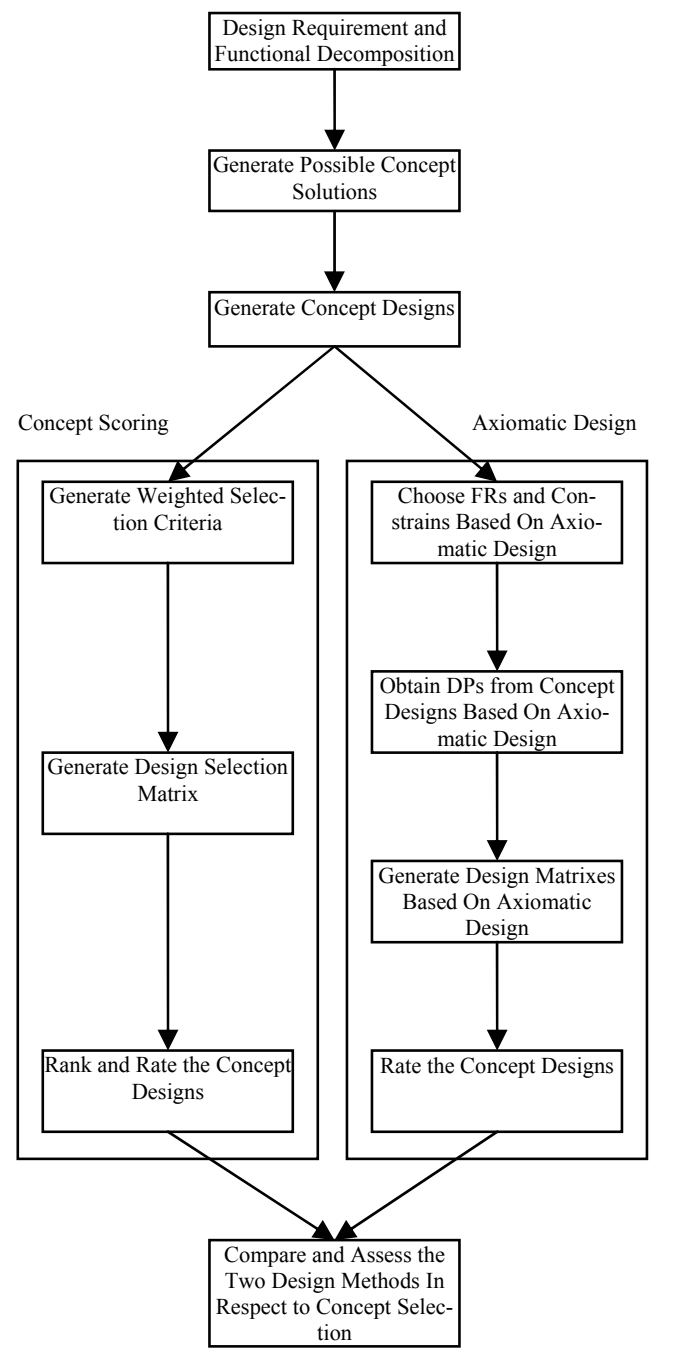

Figure 1. General Procedure

Once functional decomposition and a function diagram have been completed, concepts can be generated that realize the desired functionality. There are two main steps in generating concept designs: 1) generate possible concept solutions, and 2) combine solutions to generate concept designs. Concept solutions to the decomposed functional requirements can be generated by researching patents and technical literature, surveying targeted user-groups, benchmarking similar products, from the designer's knowledge, and/or by brainstorming. Concept designs are obtained by synthesizing a solution for all required sub-functions. 


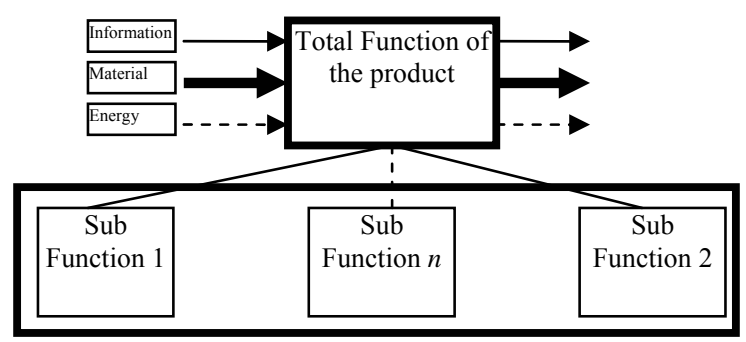

Figure 2. Traditional Methodology for Function Decomposition

\section{Preliminary Design Tasks}

The micro-pump is one of the fundamental components of micro-fluidic devices. Micro-pumps are used to deliver precisely controlled quantities, of a microliter or less, of working fluids in a variety of applications, including miniature fuel cells [15], micro cooling systems [16], biomedical analysis [17], etc. Compared with macro-sized pumps, besides their smaller size, the mechanisms for generating flow and preventing backflow are different due to the special characteristics of micro fluids. Usually, an additional basic requirement for micro-pumps is the delivery of a variety of fluids having different properties (e.g. liquid, gas). In this section, the functional decomposition of a micro-pump will be examined, solution concepts will be generated in order to facilitate unbiased choices, and designs will be proposed.

\subsection{Functional Decomposition of Micro-Pumps}

The main functions required of a micro-pump are defined and decomposed as delivery of fluid, flexibility (e.g. operate with a variety of fluids), manufacturability, features (e.g. small size), durability (e.g. long service time), and quality (e.g. high efficiency). To avoid missing creative ideas, the core function - fluid delivery - was decomposed without bias to any existing micro-pump design. A function decomposition chart for a micro-pump is shown in Figure 3.

Figure 3 shows that some requirements of micropumps, such as the ability to operate with a variety of fluids, long service time, etc., relate to every aspect of performance (represented by a dashed line). In the axiomatic design approach, these design requirements (shown in the second row of Figure 3) were considered as constraints, and the core functions (shown in the third row of Figure 3) were considered as functional requirements.

\subsection{Generation of Solution Concepts}

Solution concepts are working principles and technologies that can be used to realize decomposed functional requirements. Figure 4 shows a concept solution chart of micro-pumps. In Figure 4, the functional requirement domain (upper part of the figure) shows the core functions of micro-pumps that are decomposed based on the material stream.

Fluid enters through the inlet and pressure gradient is generated through various means of actuators and then the fluid is delivered to the outlet. The solution concept domain (lower part of Figure 4) shows the possible solutions for the corresponding sub-functions. The compatibility between the solutions can also be identified through the dashed lines between solution concepts. For example, if PZT is selected as the actuating mechanism, then one of the listed valves should be used; whereas, if rotary gears are selected as the actu-

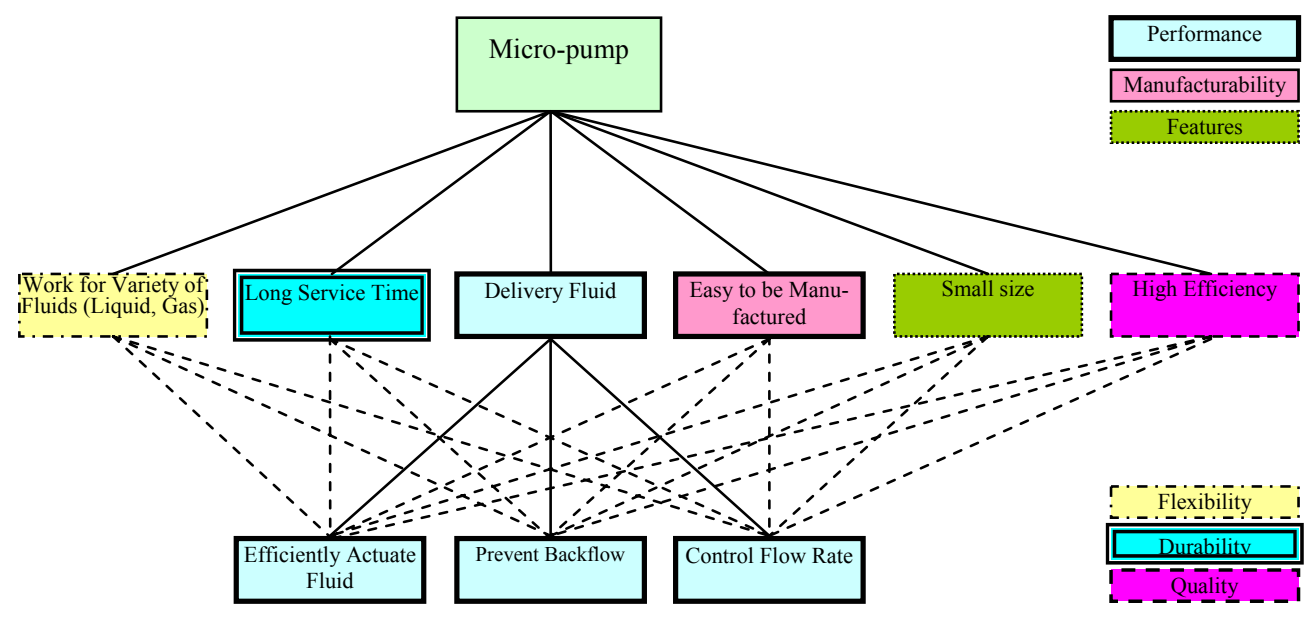

Figure 3. Function Decomposition of Micro-Pump 
Functional Requirement Domain

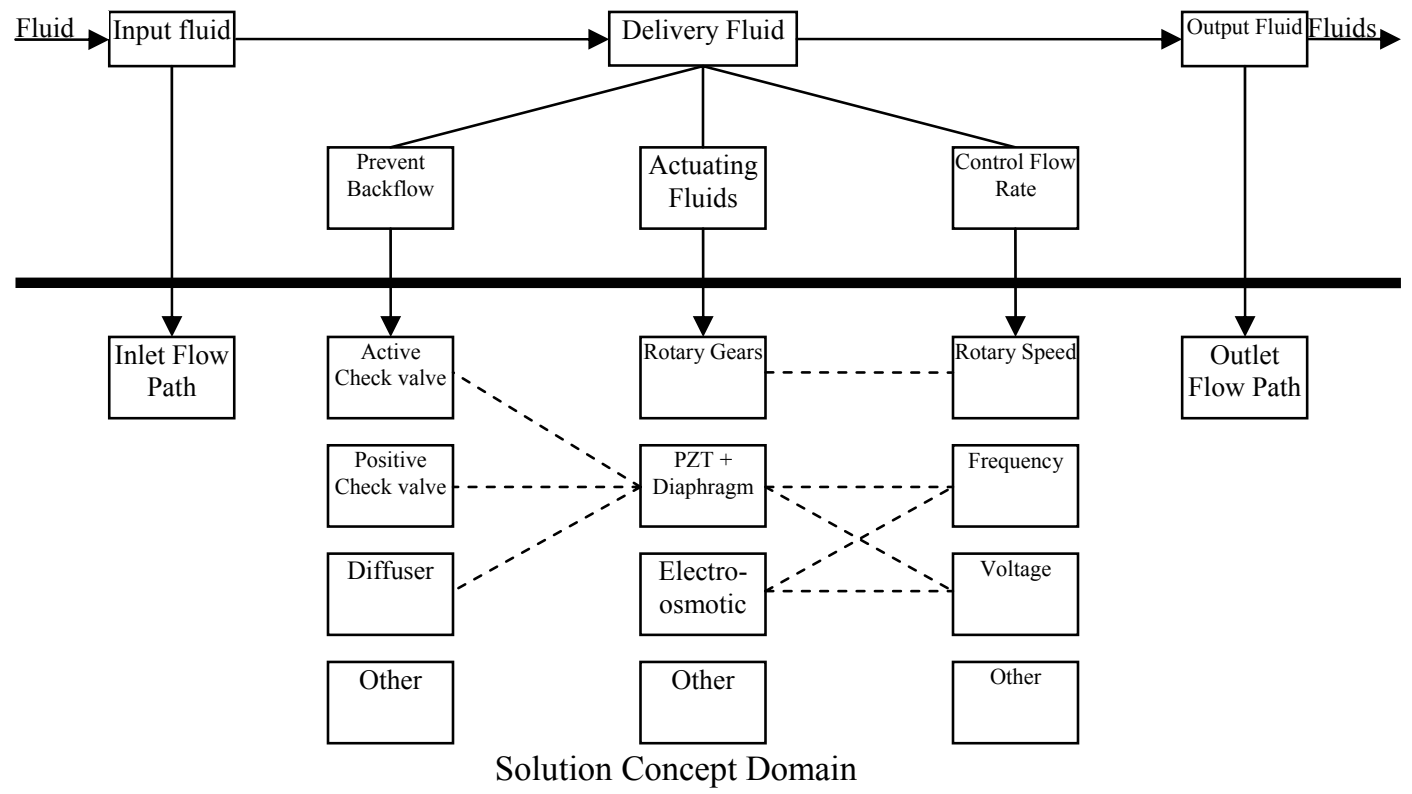

Figure 4. Concept Solution Chart

ating mechanism, no valve is needed. Figure 4 can facilitate an unbiased selection of solution concepts.

\subsection{Design Concept Generation}

Based on the concept selection chart in Figure 4, three concepts have been chosen as examples to demonstrate the evaluation of the two design selection methods. For the purpose of illustration, two similar actuating mechanisms and three totally different technologies for preventing backflow were used.

In Design Concept 1 (DC1), as shown in Figure 5, a membrane is driven by a commercially available PZT (piezoelectric transducer) disk. Two passive flap valves are integrated in the structure. When the diaphragm relaxes, fluid is sucked in through the inlet valve, while the outlet valve is closed to generate pressure gradients. When the diaphragm deforms down, fluid is pushed through the outlet valve, while the inlet valve is closed to prevent backflow. This design is simple and can be produced in a small size; and, many of the components can be fabricated using polymer materials using an injection molding process.

In Design Concept 2 (DC2), as illustrated in Figure 6, the working principle is a conventional gear pump. The gear pairs are driven by an external rotary motor. When the gear pair rotates in the direction shown in the figure, the volume of the fluid decreases in the inlet area and increases in the outlet area. The mated gears prevent the backflow. Since the size of gears is very small, the manufacturing accuracy requirements are very high. Furthermore, the external motor increases the overall size of the micro-pump.

In Design Concept 3 (DC3), as shown in Figure 7, the diaphragm is driven by a special PZT disk. In order to maintain a reasonable flow rate and take into account backflow, the deformation of the PZT is required to be larger than that needed for DC1. Both the inlet and outlet paths are integrated with a diffuser valve design. Because of pressure losses through the specially shaped inlet and outlet paths when the diaphragm deforms downward, more fluid is pushed through the outlet than the inlet, and vice versa when the diaphragm relaxes. Since the structure of this design is very simple, injection molding processes can also be used for its fabrication.
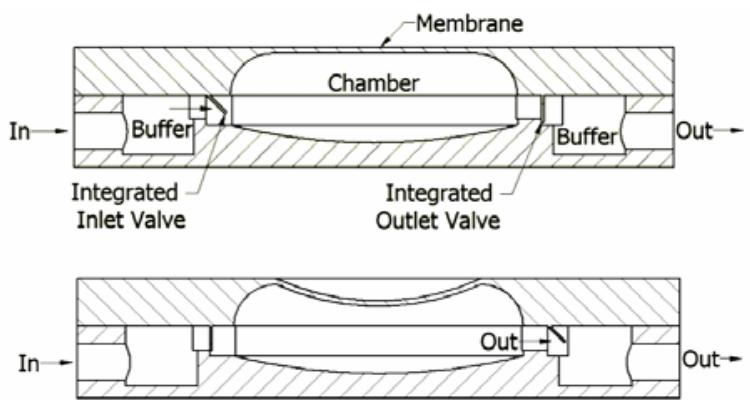

Figure 5. Design Concept 1 (DC1) 

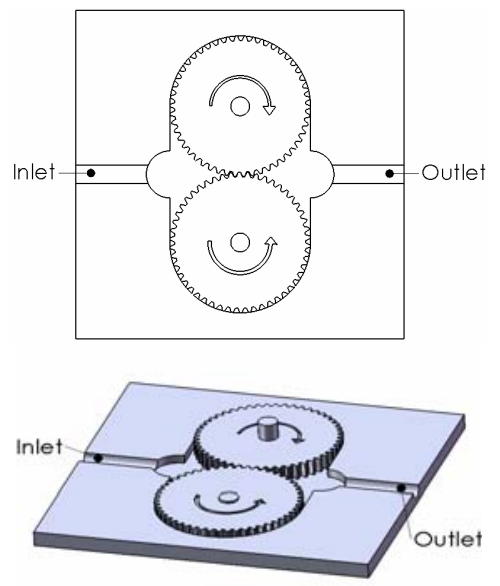

Figure 6. Design Concept 2 (DC2)

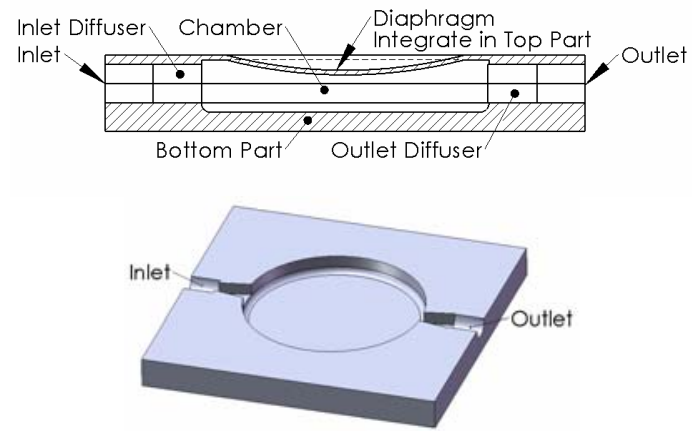

Figure 7. Design Concept 3 (DC3)

The two designs based on piezoelectric transducer actuators (CD1 and CD3), when equipped with the appropriate valve design, can deliver either liquid or gas for various applications. In DC2, the valve is actually integrated with the channel but backlash and clearance may permit backflow. However, the gear pump design can process high viscosity liquids. In the following sections, the conceptual micro-pump designs are evaluated to choose the optimal (or a "good") design using the concept scoring and axiomatic design methods.

\section{Design Concept Assessment}

The design concepts for micro-pumps are evaluated using the concept scoring and axiomatic design methods based on the selection criteria. In order to minimize bias, several designers/graduate students have evaluated the scoring and axiomatic design approaches for the designs.

\subsection{Concept Scoring Selection Method}

The concept scoring method uses a scoring matrix to evaluate concept alternatives. A reference design should be selected as the datum. Selection criteria are generated based on design requirements, which include the customers' requirements and designers' intentions.

For purpose of concept selection, the weight of the criteria can be determined subjectively by team consensus or through a designer's rational intent. A percentage is normally used to assign the weight. Design concepts are rated through a comparison to the datum design. Representative ratings are valued from 1 to 5 , indicating much worse to much better designs than the reference. Once all the concepts are rated, a total score for each design is calculated using the following formula:

$$
\text { Total Score }=\sum_{1}^{n} w_{n} \times d_{n}
$$

where, $n$ is the count of selection criteria, $w_{n}$ is the weight of $n$th criteria, and $d_{n}$ is the rating of the $n$th criteria. The overall performances of the alternative designs are ranked based on their total score. The relative strengths and weaknesses of alternative concepts can be identified by investigating the concept scoring matrix. The improved concept design can be obtained by adjusting or combining different concept designs.

To evaluate the micro-pump designs using concept scoring, the development of criteria weights that were reasonable and aligned with priorities was required. For example, the prevention of backflow and a small footprint are important requirements for the micropumps. Any pump will tend to have some backflow due to backpressure; and, the ability to prevent backflow is what actually constitutes pumping, so preventing backflow is imperative. Since most micro-pumps are integrated into a micro-electro-mechanical system (MEMS) and often used for portable electronics, a small size is important; whereas, the ability to operate with various fluids is relatively less important. Representative weights for these criteria are listed in Table 1.

The design concepts were rated by several graduate students with design experience, and their assessments were averaged and are shown in Table 2. According to Box [18], "It has further been shown that the wider team - even if they do not play an active role in concept generation - can aid in the quality of output by acting as a listening device." The design concepts were compared to DC1, which was selected as the reference (datum) design. 
Table 1. Selection Criteria and Weights

\begin{tabular}{|c|c|c|}
\hline \multirow{2}{*}{ Number } & Selection Criteria & Weight \\
\hline C1 & Working for Variety of Fluid & $10 \%$ \\
\hline C2 & Long Service Time & $10 \%$ \\
\hline C3 & Actuate Fluid & $5 \%$ \\
\hline C4 & Prevent Backflow & $25 \%$ \\
\hline C5 & Effectively Control Flow Rate & $10 \%$ \\
\hline C6 & Easy to be Manufactured & $10 \%$ \\
\hline C7 & Small Size & $20 \%$ \\
\hline C8 & High Efficiency & $10 \%$ \\
\hline
\end{tabular}

From the concept scoring matrix (design selection matrix) in Table 2, it can be seen that DC1 was the most preferable choice, while DC2 was the least desirable. Design Concept 2 was assessed to have a longer service time, better fluid actuation, and more effective flow rate control than DC1. However, DC2 violated the space requirements, due to the external motor. It can also be seen that DC3 was better than DC1 with respect to length of service time, manufacturability and small size; however, these strengths did not make up for their other deficiencies. The concept scoring method analysis, therefore, shows that the design concept with the PZT actuator and the flap valves provides the optimal design compared with the others.

\subsection{Axiomatic Design Selection Method}

The axiomatic design selection method mathematically maps criteria to derive a good design based on axioms [12]. As a systematic design method, axiomatic design treats the design as a mapping process between the functional domain (functional requirements or FRs) and the physical domain (design parameters or DPs), as shown in Figure 8. The mathematical representation of the design matrix operation is:

$$
\{\mathrm{FRs}\}=[\mathrm{A}]\{\mathrm{DPs}\}
$$

where, FRs are defined as the minimum set of independent requirements that completely characterize the functional needs of the product design in the functional domain [12]. DPs are defined as the key variables that characterize the physical entity created by the design process and that fulfill the FRs. The design matrix, A, shows the relationship between FRs and DPs. In axiomatic design, total design requirements are defined by either FRs or constraints. Constraints are defined as bounds on an acceptable solution, often having a limiting effect on design.

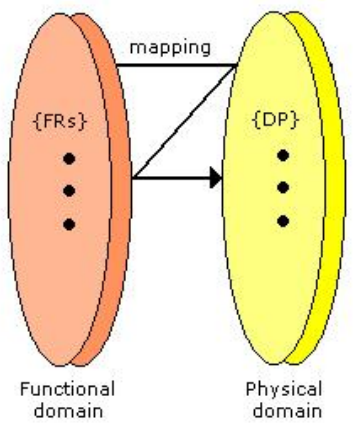

\section{Figure 8. Functional Domain to Physical Domain [12]}

According to the axiomatic design method, designs are considered to be good when the independence and information axioms are satisfied. A good design occurs when the FRs are independent of each other (e.g. uncoupled as shown in Figure 9(a) or a decoupled matrix as shown in Figure 9(b)); and, likewise, a "bad" design arises when the matrix is coupled (Figure 9(c)).

Table 2. Concept Scoring Matrix

\begin{tabular}{|c|c|c|c|c|c|c|c|}
\hline \multirow[b]{2}{*}{ Criteria } & \multirow[b]{2}{*}{ Weight } & \multicolumn{2}{|c|}{$\begin{array}{c}\text { Design Concept } 1 \\
\text { (reference) } \\
\text { (PZT and Flap Valve) }\end{array}$} & \multicolumn{2}{|c|}{$\begin{array}{c}\text { Design Concept } 2 \\
\text { (Gear Pump) }\end{array}$} & \multicolumn{2}{|c|}{$\begin{array}{c}\text { Design Concept } 3 \\
\text { (PZT and Valveless } \\
\text { Diffuser) }\end{array}$} \\
\hline & & Rating & Score & Rating & Score & Rating & Score \\
\hline $\mathrm{C} 1$ & $10 \%$ & 3 & 0.3 & 1.5 & 0.15 & 2.25 & 0.225 \\
\hline $\mathrm{C} 2$ & $10 \%$ & 3 & 0.3 & 4.25 & 0.425 & 4.25 & 0.425 \\
\hline $\mathrm{C} 3$ & $5 \%$ & 3 & 0.15 & 4.25 & 0.2125 & 3 & 0.15 \\
\hline $\mathrm{C} 4$ & $25 \%$ & 3 & 0.75 & 3 & 0.75 & 1.5 & 0.375 \\
\hline $\mathrm{C} 5$ & $10 \%$ & 3 & 0.3 & 4 & 0.4 & 1.5 & 0.15 \\
\hline C6 & $10 \%$ & 3 & 0.3 & 1.5 & 0.15 & 4.5 & 0.45 \\
\hline $\mathrm{C} 7$ & $20 \%$ & 3 & 0.6 & 1.75 & 0.35 & 4.5 & 0.9 \\
\hline $\mathrm{C} 8$ & $10 \%$ & 3 & 0.3 & 3.25 & 0.325 & 1.75 & 0.175 \\
\hline \multicolumn{2}{|c|}{ Total Score } & & 3 & & 2.8 & & 2.9 \\
\hline \multicolumn{2}{|c|}{ Rank } & & 1 & & 3 & & 2 \\
\hline
\end{tabular}


In many real designs, the values in the design matrix are nonlinear, due to the nonlinear relationships between FRs and DPs. In this situation, an uncoupled design matrix may become coupled when the operating range of certain DPs is shifted. In order to reflect this situation, reangularity $(\mathrm{R})$ and semangularity $(\mathrm{S})$ was used to measure quantitatively the sensitivity of FRs to changes in DPs and the independence of FRs, respectively [12]. However, this quantitative method is not easy to use.

$$
\left\{\begin{array}{l}
F R 1 \\
F R 2 \\
F R 3
\end{array}\right\}=\left[\begin{array}{ccc}
X & 0 & 0 \\
0 & X & 0 \\
0 & 0 & X
\end{array}\right]\left\{\begin{array}{l}
D P 1 \\
D P 2 \\
D P 3
\end{array}\right\}
$$

(a) Uncoupled Design Matrix

$$
\left\{\begin{array}{l}
F R 1 \\
F R 2 \\
F R 3
\end{array}\right\}=\left[\begin{array}{ccc}
X & X & X \\
0 & X & X \\
0 & 0 & X
\end{array}\right]\left\{\begin{array}{l}
D P 1 \\
D P 2 \\
D P 3
\end{array}\right\}
$$

(b) Decoupled Design Matrix

$$
\left\{\begin{array}{l}
F R 1 \\
F R 2 \\
F R 3
\end{array}\right\}=\left[\begin{array}{ccc}
X & 0 & X \\
0 & X & X \\
X & 0 & X
\end{array}\right]\left\{\begin{array}{l}
D P 1 \\
D P 2 \\
D P 3
\end{array}\right\}
$$

(c) Coupled Design Matrix

\section{Figure 9. Axiomatic Design Matrices}

By examining Suh's axiomatic design theorems and corollaries [12], seven criteria have been formulated that can be simply applied to judge between good and bad design concepts after a design matrix is obtained:

C1. A design that does not avoid the design constraints is better than one that avoids them.
C2. A less coupled design is better than a more coupled design.

C3. A design with less components or features is better than one with more components or features.

C4. A design that uses more standardized parts is better than one that uses fewer standardized parts.

C5. A design with a square design matrix is better than a design with a non-square design matrix (minimize redundancy).

C6. A design with less tolerance requirements is better than one with high tolerance requirements.

C7. A design with less sensitivity to coupled (offdiagonal) FRs to the corresponding DPs is better than one with more sensitivity to coupled DPs.

The axiomatic design method, as applied to the analysis of the micro-pump designs, first requires the formulation of independent FRs, a non-trivial task. It is followed by determining DPs from concept designs. The design matrix, A, can then be evaluated based on the above seven criteria.

Three functional requirements were selected based on Figure 5, and the design parameters were selected based on Figure 6 . In order to make it easier to compare the design concepts and explain the application of the concept to the proposed axiomatic design selection criteria, all of the functional requirements have been intentionally decomposed to be the same. The design matrix mapping of FRs to Dps, shown in the following

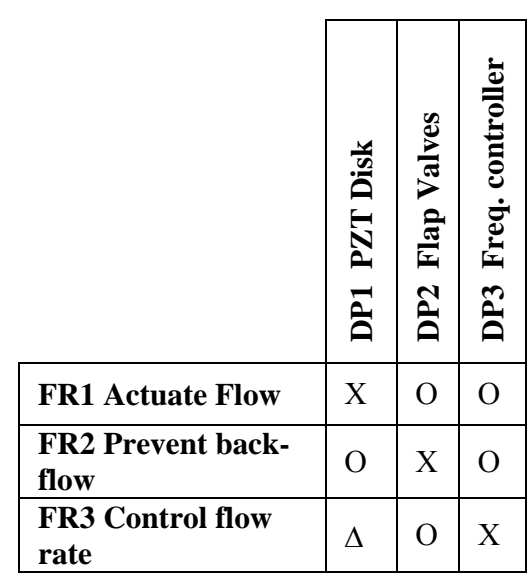

Figure 10: Design Matrix for Concept Design 1 (DC1)

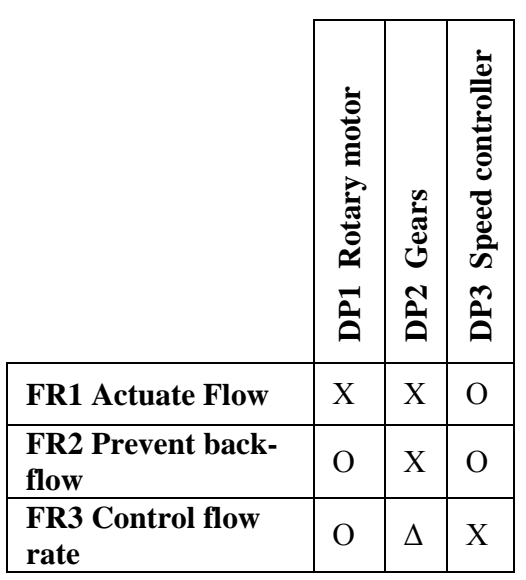

Figure 11: Design Matrix for Concept Design 2 (DC2) 


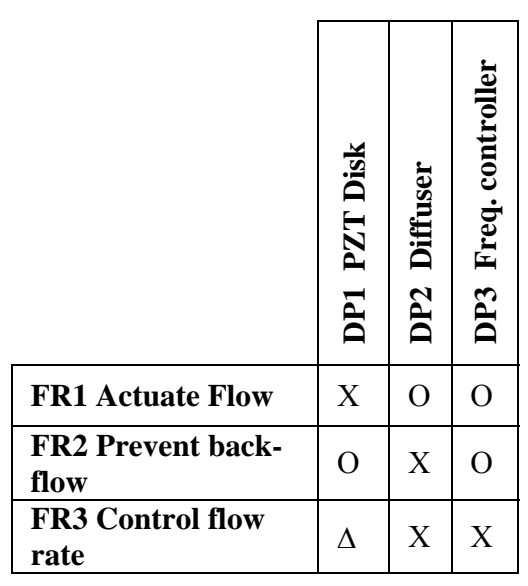

Figure 12: Design Matrix for Concept
Design 3 (DC3)

figures, are: $\mathrm{X}$ - strongly related, $\Delta$ - weakly related, and $\mathrm{O}$ - very weak to no relation.

The design matrix for DC1 is shown in Figure 10. The design matrix is a weakly uncoupled design, since the PZT disk slightly influences the control of flow rate [19]. The design matrix for DC2, as shown in Figure 11 , is a coupled design since the dual gears influence actuation of the flow rate. Furthermore, leakage may occur caused by the backlash between the gears and the clearance between gears. The axiomatic design evaluation for DC3, as shown in Figure 12, also exhibits a similar trend as DC1, with the exception of where the diffuser design strongly influences flow rate because backflow cannot be prevented. Therefore, the third design is a decoupled design, which is acceptable but not as good as the first design.

The internal technical conflicts of each design were also identified. Criteria $\mathrm{C} 1$ indicates that $\mathrm{DC} 2$ is poor compared to DC1 and DC3, as the gear pair is not applicable for pumping gas. Criteria $\mathrm{C} 2$ indicates that DC1 is better than DC3 because it is less coupled. Criteria $\mathrm{C} 3$ indicates that $\mathrm{DC} 3$ is better than $\mathrm{DC} 1$, because the diffuser valve is integrated into the flow path. Criteria $\mathrm{C} 4$ indicates that $\mathrm{DC} 1$ is better than DC3, because DC1 uses a commercially available PZT disk, while DC3 uses a special PZT due to the large deformation requirement. Criteria C6 indicates that $\mathrm{DC} 3$ is better than both $\mathrm{DC} 1$ and $\mathrm{DC} 2$, because the diffuser design has a smaller tolerance requirement compared with the high tolerance requirements on the backlash of gear in DC2 and the flap valve contact surface on DC1. Criteria C7 indicates that DC1 is better than DC3, because the dynamic characteristic of the flap valve is not as sensitive as that of a diffuser [20].
Based on the criteria and design matrix analyses in the axiomatic design approach, the evaluation of the micro-pump designs also exhibit a similar trend as the concept scoring method, by selecting DC1 as the most suitable choice, followed by DC3 and then DC2.

\section{Comparison of Concept Scoring and Axiomatic Design Methods}

The two methods for evaluating micro-pumps have led to the same conclusion and provide effective tools to aid designers to select the optimal or good design concepts. Their comparison gives advantages and disadvantages of the individual methods, so that designers can choose the appropriate technique. Table 3 outlines the comparison of the methods, and their ability to meet each metric is rated from 0 (poor) to 2 (good). The metrics for evaluating the selection methods are as follows:

M1. Ability to treat non-technical requirements, such as aesthetics, cost and other closely related design requirements, which were classified as constraints in the axiomatic design.

M2. Feasibility of the design.

M3. Ability to aid improved concept design.

M4. Reliability (objective vs. subjective).

M5. Ease of use.

M6. Agility for changing environments.

Table 3. Comparison of the Concept Scoring and Axiomatic Design Methods with Respect to Concept Selection

\begin{tabular}{|c|c|c|c|}
\hline Metrics & $\begin{array}{c}\text { Concept } \\
\text { Scoring }\end{array}$ & $\begin{array}{c}\text { Axiomatic } \\
\text { Design }\end{array}$ & Reasons \\
\hline $\begin{array}{c}\text { M1 - Non- } \\
\text { Technical }\end{array}$ & 2 & 0 & R1 \\
\hline M2 - Feasibility & 0 & 2 & R2 \\
\hline M3 - Improved & 1 & 1 & R3 \\
\hline M4 - Reliability & 0 & 2 & R4 \\
\hline M5 - Ease of Use & 2 & 0 & R5 \\
\hline M6 - Agility & 2 & 0 & R6 \\
\hline Total & $\mathbf{7}$ & $\mathbf{5}$ & \\
\hline
\end{tabular}

The reasons for the scoring of Table 3 are detailed in the following paragraphs.

R1: Although it is more rational to define nontechnical design requirements as constraints (as done in the axiomatic design), analysis of constraints to the design parameters are not straightforward, especially when comparing alternative designs. This is due to the reality that detailed information of each design concept is not available. In the micro-pump designs, the 
only constraint applied for concept selection using the axiomatic design method was the ability to operate with a variety of fluids and size limitations. Other design requirements, such as long service life and efficiency, were not evaluated because details, such as fatigue analysis, the manufacturing process, power consumption calculations, and material selections, were not taken into consideration. However, instead of ignoring these design requirements, the concept scoring method was more practical in dealing with nontechnical design requirements, during the concept selection stage.

$\mathrm{R} 2$ : The information in the axiomatic design matrix can show the complexity of the concept designs and provide the influences of design parameters on the functional requirements. Coupled matrix designs are harder to achieve; and, when one of the design parameter becomes inactive, the rest of design is affected. For example, it is easier to choose the dimensions of a flap valve than a diffuser valve design, since the dimension of the diffuser valve strongly and simultaneously influences two requirements - minimized backflow and controlled flow rate. This means that, even if the flow rate is controlled, the backflow may be poor. The concept scoring method did not provide solutions in this aspect.

R3: The concept scoring method can help designers identify the strengths and weaknesses of alternative designs; and, by combining of the advantages of different designs, a designer can achieve an improved design. Axiomatic design identifies compatibility of design parameters toward fulfilling functional requirements, e.g. DPs that lead to less coupled FRs make a better design.

R4: From the process of using the two design methods, it can seen that the concept scoring method relies heavily on biased personal judgements in both weighting and scoring, while the axiomatic design method relies more on technical knowledge. Thus, axiomatic design is more objective compared with the concept scoring method.

R5: The concept scoring method is easier to use, because it is easy to determine the advantages and disadvantages of designs in achieving the design requirements. Ranking the designs through sum of scores is easily understood. Axiomatic design can only effectively come up with a good or bad design depending on the relationship between functional requirements and design parameters. However, the selection of independent functional requirements and design parameters is challenging, and making a coupled design to an uncoupled or decoupled design requires several iterations before one can be satisfied with the final set.

R6: Rapidly changing market demands require designs to be agile in order to adapt to new needs. In their empirical study of the automotive industry, Almefelt et al. [21] have found that requirements are changed, added, and reprioritized throughout the course of the development process. The concept scoring method is more efficient, because when functional requirements are changed, only the weighting information needs to be adjusted to reflect the change; and, most of the scoring information can still be used. However, for axiomatic design, when a functional requirement is changed, new design parameters must be determined to satisfy the new set of FRs; and, all the information in the design matrix cannot be reused.

The two methods provide effective means to evaluate early stage conceptual designs. The concept scoring method is easy to use and agile compared with the axiomatic design. The axiomatic design approach shows advantages in feasibility by decoupling the functional requirements with the design parameters, so that when a problem rises in the case of uncoupled designs, only one design parameter can be changed to satisfy the overall design.

\section{Conclusions and Future Work}

The two design methods have their own strengths and weaknesses to aid designers in achieving optimal or good design concept selection. The concept scoring method is an efficient and easy-to-use tool, and it can address design requirements more extensively. The axiomatic design method, unlike concept scoring, cannot give an overall ranking of design alternatives, but it can identify internal technical conflicts. Concept scoring is more subjective, while axiomatic design is more objective. Axiomatic design is difficult to use, especially in finding a set of independent FRs and DPs [12]. Moreover, interpreting the axiomatic design matrix is not straightforward when the design gets more complex, since the design is more likely to become coupled between FRs and DPs. Even though both methods provide optimal design selections, as shown with the micro-pump examples, the performance of concept scoring is more appropriate to that of axiomatic design, when comparing the various designs with the selection criteria.

For recommended future work, the two methods should be investigated as to their capability of integrating the advantages of each method. For example, the concept scoring method can provide ease of use 
and agility, and can address aesthetics and cost. The axiomatic design method can provide mathematical robustness to the design by uncoupling the requirements and design parameters. One approach to achieve this integration will be by providing weights between FRs and DPs, in order to find the degrees of influence [22]. This will provide ranking of the designs and minimize redundancy through matrix manipulations. A combined method will also allow for a more effective way to select appropriate FRs and DPs, instead of an ad-hoc approach. Further, an integrated concept scoring and axiomatic design method may be enhanced to assess technical feasibility, tolerance requirements, usage of standard parts, etc.

\section{References}

[1] K.T. Ulrich, and S.D. Eppinger, Product Design and Development $2^{\text {nd }}$ ed., McGraw-Hill, New York, 2000.

[2] J. Nevins and D. Whitney, Concurrent Design of Products and Processes, McGraw-Hill, New York, 1989.

[3] H.J. Wassenaar and W. Chen, "An Approach to Decision-Based Design", Proceedings of ASME DETC'01, September 9-12, 2001, Pittsburgh, Pennsylvania, USA.

[4] S. Pugh, Total Design - Integrated Methods for Successful Product Engineering, Addison-Wesley, MA, 1990.

[5] D.L. Thurston and J.V. Carnahan, "Fuzzy Ratings and Utility Analysis in Preliminary Design Evaluation of Multiple Attributes", Journal of Mechanical Design, Vol. 114, 1992, pp. 648-658.

[6] S.H. Yeo, M.W. Mak and A.P. Balon, "Analysis of Decision-making Methodologies for Desirability Score of Conceptual Design", Journal of Engineering Design, Vol. 15, 2004, pp. 195-208.

[7] A.M. King and S. Sivaloganathan, "Development of a Methodology for Concept Selection in Flexible Design Strategies", Journal of Engineering Design, Vol. 10, 1999, pp. 329-349.

[8] T.K. See and K. Lewis, "Multi attribute Decision Making Using Hypothetical Equivalents", Proceedings of ASME DETC'02, September 29 - October 2, 2002, Montreal, Canada.

[9] J. Hauser and D. Clausing, "The House of Quality", Harvard Business Review, May 1988, pp. 67-73.
[10] M. Salonen and M. Perttula, "Utilization of Concept Selection Methods - A Survey of Finnish Industry", Proceeding of IDETC/CIE 2005, September 24-28, 2005, Long Beach, California USA.

[11] J. Wang, "Improved engineering design concept selection using fuzzy sets", International Journal of Computer Integrated Manufacturing, Vol. 15, No. 1, 2002, pp. 18-27.

[12] N.P. Suh, The Principles of Design, Oxford University Press, New York, 1990.

[13] M. Hu and J. Pieprzak, "Using axiomatic design to improve conceptual design robustness in Design for Six Sigma (DFSS) methodology", International Journal of Six Sigma and Competitive Advantage (IJSSCA), Vol. 1, No. 3, 2005.

[14] Y. Umeda and T. Tomiyama, "Functional Reasoning in Design”, IEEE Expert, March 1997, pp. 42-48.

[15] T. Zhang and Q. Wang, "Valveless Piezoelectric Micropump for Fuel Delivery in Direct Methanol Fuel Cell (DMFC) Devices", Journal of Power Sources, Vol. 140/1, 2005, pp. 72-80.

[16] J. Schutze, H. Ilgen and W.R. Fahrner, "An integrated micro cooling system for electronic circuits", Industrial Electronics, IEEE Transactions, Vol. 48, Iss. 2, Apr. 2001, pp. 281-285.

[17] T. Thorsen, S.J. Maerkl and S.R. Quake, "Microfluidic large-scale integration", Science 298:5, 2002, pp. $80-84$.

[18] G. Box, "Good quality costs less. How come?" Quality Engineering, Vol. 3/1, 1990.

[19] S. Li and S. Chen, "Analytical analysis of a circular PZT actuator for valveless micropumps", Sensors and Actuators A, Vol. 104, 2003, pp. 151-161.

[20] J. Laser and J.G. Santiago, "A review of micropumps", Journal of Micromechanics and Microengineering, 14, 2004, pp. 35-64.

[21] L. Almefelt, F. Andersson, P. Nilsson and J. Malmqvist, "Exploring requirements management in the automotive industry", Proceedings of ICED'03, August 19-21, 2003, Stockholm, Sweden, pp. 14.

[22] T. Freiheit, S.S. Park and V. Giuliani, "An Integrated Design Methodology", Proceedings of the Second CDEN International Conference on Design Education, Innovation, and Practice, Kananaskis, Alberta, Canada, July 18-20, 2005. 